\title{
Re-evaluation of the parton distribution of strange quarks in the nucleon
}

\section{H. E. Jackson*}

Physics Division, Argonne National Laboratory, Argonne, Illinois 60439-4843, USA

E-mail: haleanl.gov

The recently published final data on the multiplicities of charged kaons in deep-inelastic scattering from the HERMES experiment has been used to re-evaluate the earlier reported extraction of parton distributions of strange quarks in the proton. In the measured range of $x$ the strength of the polarization-averaged PDF $\mathrm{S}(\mathrm{x})$ is substantially less than obtained earlier, but the shape is similar, i.e., the momentum densities are softer than previously assumed. Within the precision of the measurement, no significant strength is observed above $x=0.1$.

On behalf of the HERMES collaboration

PACS: 13.60.-r, 13.88.+e, 14.20.Dh, 14.65.-q

XXI International Workshop on Deep-Inelastic Scattering and Related Subject-DIS2013,

22-26 April 2013

Marseilles, France

${ }^{*}$ Speaker. 


\section{Introduction}

In 2008 HERMES published the results of an "isoscalar" extraction in leading logarithmic order (LO) in the strong coupling constant of quantum chromodynamics of the momentum and helicity density distributions of the strange sea in the nucleon from the charged-kaon production in deep-inelastic scattering (DIS) on the deuteron [1]. The shape of the momentum distribution was observed to be softer than that of the average of the $\bar{u}$ and $\bar{d}$ quarks. HERMEs has since finalized the extraction of multiplicites for charged kaons in semi-inclusive deep-inelastic positron scattering (SIDIS) [2] using a more refined procedure for removing the effects of spectrometer acceptance, radiative processes, and kinematic smearing. The new values for the multiplicities of charged kaons, shown in Fig. 1 as a function of $x$, the Bjorken scaling variable, are substantially larger in the region $x>0.1$ than those in the data used in 2008 to extract the PDFs for strange quarks in the proton. Because the high $x$ region is, in leading order (LO), dominated by the fragmentation of nonstrange quarks, the use of the new values in the extraction of the momentum parton distribution function (PDF), substantially decrease the estimate of the contribution of strange quarks to the charged kaon multiplicity. The results of the updated extraction are reported here.

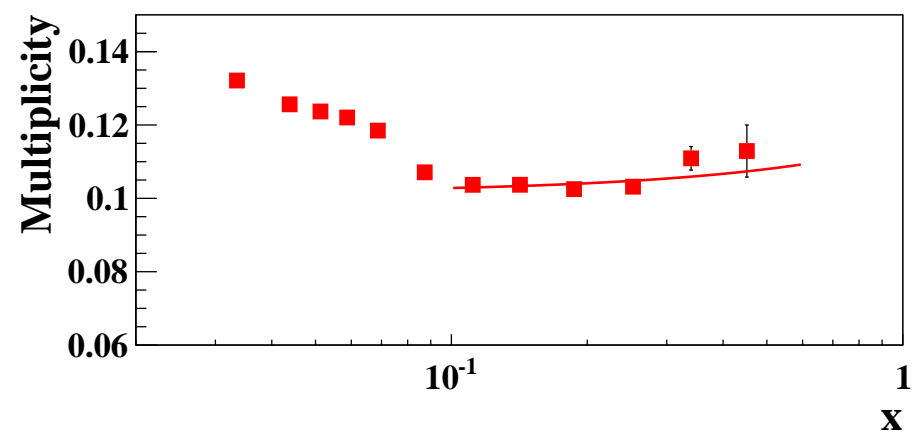

Figure 1: The multiplicity corrected to $4 \pi$ of charged kaons in SIDIS from a deuterium target as a function of Bjorken $x$ [2]. The solid curve is a first degree polynominal fit to the data above $x>0.1$ (see text).

\section{Extraction of $S(x)=s(x)+\bar{s}(x)$}

In the isoscalar method [1], the momentum distribution of the strange quark sea is extracted in LO from spin-averaged $K^{ \pm}$multiplicities measured in the scattering of polarized positrons by a polarized deuterium target. For the isoscalar deuteron, in LO these observables depend on the PDFs $Q(x) \equiv u(x)+\bar{u}(x)+d(x)+\bar{d}(x)$ and $S(x) \equiv s(x)+\bar{s}(x)$. The fragmentation process can be described by fragmentation functions that have no isospin dependence. Aside from isospin symmetry between proton and neutron, the only symmetry assumed is charge-conjugation invariance in fragmentation. In LO, the inclusive unpolarized (U) electron scattering cross section in terms of the parton distributions $Q(x) \equiv u(x)+\bar{u}(x)+d(x)+\bar{d}(x)$ and $S(x) \equiv s(x)+\bar{s}(x)$ takes the form

$$
\frac{\mathrm{d}^{2} N^{D I S}(x)}{\mathrm{d} x \mathrm{~d} Q^{2}}=\mathscr{K}_{U}\left(x, Q^{2}\right)[5 Q(x)+2 S(x)]
$$


where $\mathscr{K}_{U}\left(x, Q^{2}\right)$ is a kinematic factor containing the hard scattering cross section. The weak logarithmic dependence of the PDFs on $-Q^{2}$, the squared four-momentum of the exchanged virtual photon, has been suppressed for simplicity. Applying the same LO formalism to the SIDIS cross section for charged kaon production, irrespective of charge, hereafter designated as $K$ gives

$$
\frac{\mathrm{d}^{2} N^{K}(x)}{\mathrm{d} x \mathrm{~d} Q^{2}}=\mathscr{K}_{U}\left(x, Q^{2}\right)\left[Q(x) \int \mathscr{D}_{Q}^{K}(z) d z+S(x) \int \mathscr{D}_{S}^{K}(z) d z\right],
$$

where $z \equiv E_{h} / v$ with $v$ and $E_{h}$ the energies of the virtual photon and of the detected hadron in the target rest frame, $\mathscr{D}_{Q}^{K}(z) \equiv 4 D_{u}^{K}(z)+D_{d}^{K}(z)$ and $\mathscr{D}_{S}^{K}(z) \equiv 2 D_{s}^{K}(z)$. The fragmentation function $D_{q}^{K}(z)$ describing the number density of charged kaons from a struck quark of flavor $q$ is integrated over the measured range of $z$. Combining Eqs. $(2.1,2.2)$ and neglecting the term $2 S(x)$ compared to $5 Q(x)$, it follows immediately that

$$
S(x) \int \mathscr{D}_{S}^{K}(z) d z \simeq Q(x)\left[5 \frac{\mathrm{d}^{2} N^{K}(x)}{\mathrm{d}^{2} N^{D I S}(x)}-\int \mathscr{D}_{Q}^{K}(z) d z\right] .
$$

Eq. 2.3 is the basis for the extraction of the quantity $S(x) \int D_{S}^{K}(z) d z$.

Since both $S(x)$ and the quantity $\int D_{S}^{K}(z) d z$ are unknown, the basic observable in the extraction is the product $S(x) \int D_{S}^{K}(z) d z$. In the region $x>0.1$ the contribution of strange quark fragmentation to the kaon multiplicity falls more rapidly with increasing $x$ than that of the nonstrange quarks. It follows that in LO the slope of the multiplicity will be negative with increasing $x$ until the strange quark contribution becomes negligible, at which point the LO multiplicity becomes identically 0.2 $\int \mathscr{D}_{Q}^{K}(z)$. Therefore, the flattening of the kaon multiplicity with increasing $x$ above 0.1 , as is present in the data shown in Fig. 1, signals that $S(x)$ is negligible in this region and that the integral $\int \mathscr{D}_{Q}^{K}(z)$ can be extracted directly from the multiplicity values in this $x$ range. To account for any residual dependence on $Q^{2}$ or equivalently on $x$, the multiplicity for $x>0.1$ was fitted to a first degree polynomial yielding the result that $d N^{K}(x) / d N^{D I S}(x)=0.1015 \pm 0.0017+(0.01294 \pm 0.010) \cdot \mathrm{x}$, as shown by the solid curve in Fig. 1 . In the region near $x=0.13$ where $Q^{2} \approx 2.5$ this fit gives the result $\int_{0.2}^{0.8} \mathscr{D}_{Q}^{K}(z, x) d z=0.506 \pm 0.010$, in fair agreement with the value $0.435 \pm 0.044$ obtained for $Q^{2}=2.5 \mathrm{GeV}^{2}$ from the most recent global analysis of fragmentation functions [3]. The weak $\mathrm{x}$ dependence in the fit is consistent with the $Q^{2}$ dependence of the data from the global analysis.

The extracted quantity $\int_{0.2}^{0.8} \mathscr{D}_{Q}^{K}(z, x) d z$ was then used together with values of $Q\left(x, Q^{2}\right)$ from CTEQ6L and the measured multiplicities in 2.3 to obtain the product $S(x) \int \mathscr{D}_{S}^{K}(z) d z$. A small iterative correction was made to account for the neglect of the $2 S(x)$ term in Eq. 2.1. The result for the product together with a fit of the form $x^{-a_{1}} e^{-x / a_{2}}(1-x)$ is shown in Fig. 2. The quantity plotted in this figure is the basic observable in the HERMES measurement. In the region $x<0.1$ the values of the product are substantially smaller than those reported in Ref. [1].

The fit to the final HERMES multiplicities resulting from summing the non-strange and strange quark contributions is presented in Fig. 3. Included in that figure is the LO prediction obtained with CTEQ6L PDFs and fragmentation functions from [3]. Clearly, the CTEQ6L PDFs yield a poor representation of the kaon multiplicities. As was observed in Ref. [1], the improved fit (continuous curve in Fig. 3) to the multiplicity is an indication that the actual distribution of $S(x)$ is substantially different from the average of those of the nonstrange antiquarks. To explore this point, the HERMES result for $S(x) \int \mathscr{D}_{S}^{K}(z) d z$ has been evolved to $Q_{0}^{2}=2.5 \mathrm{GeV}^{2}$. The $Q^{2}$ evolution factors were 


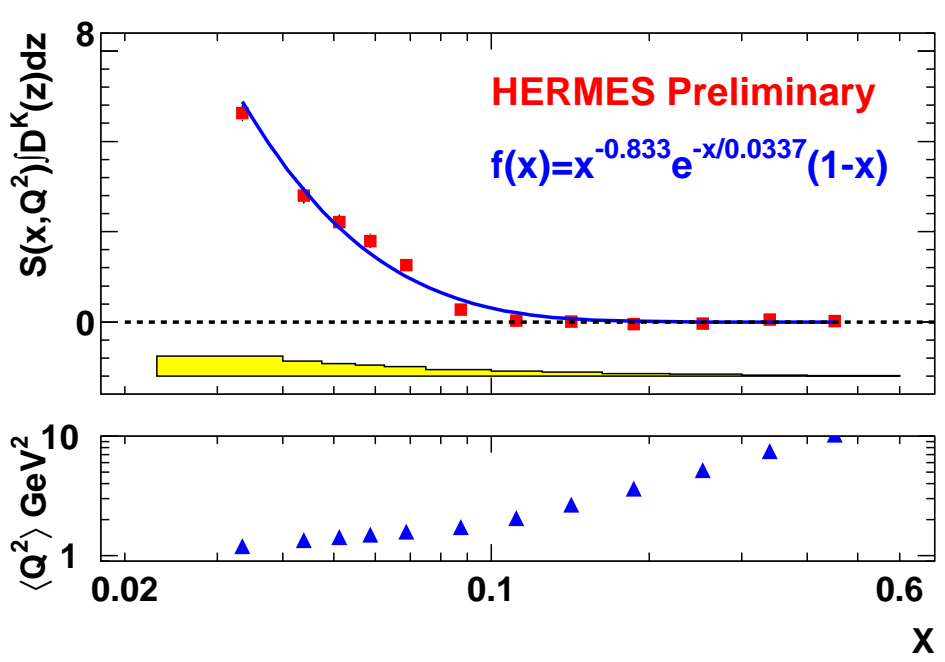

Figure 2: The strange fragmentation product $S\left(x, Q^{2}\right) \int \mathscr{D}_{S}^{K}(z) d z$ obtained from the measured multiplicity for charged kaons at the $\left\langle Q^{2}\right\rangle$ for each bin. The curve is a least squares fit of the form $x^{-0.834} e^{-x / 0.0337}(1-x)$. The band represents systematic uncertainties. The values of $\left\langle Q^{2}\right\rangle$ for each $x$ bin are shown in the lower panel.

taken from CTEQ6L and the fragmentation function compilation given in [3]. Consideration of corrections to the evolution due to higher twist contributions is not necessary, since higher twist effects are expected to be significant [4] only for larger values of $x$ where the extracted distribution of $x S(x)$ vanishes.

In order to compare the HERMES result for $S(x) \int \mathscr{D}_{S}^{K}(z) d z$ with existing estimates of $\mathrm{S}(\mathrm{x})$ in the literature it is necessary to assume a value for $\int \mathscr{D}_{S}^{K}(z) d z$. The distribution of $x S(x)$ was obtained from $S(x) \int \mathscr{D}_{S}^{K}(z) d z$ by dividing by $\int \mathscr{D}_{S}^{K}(z) d z=1.27 \pm 0.13$, the value at $Q^{2}=2.5 \mathrm{GeV}^{2}$ given in [3]. The results are presented in Fig. 4. The normalization of the HERMES points is determined

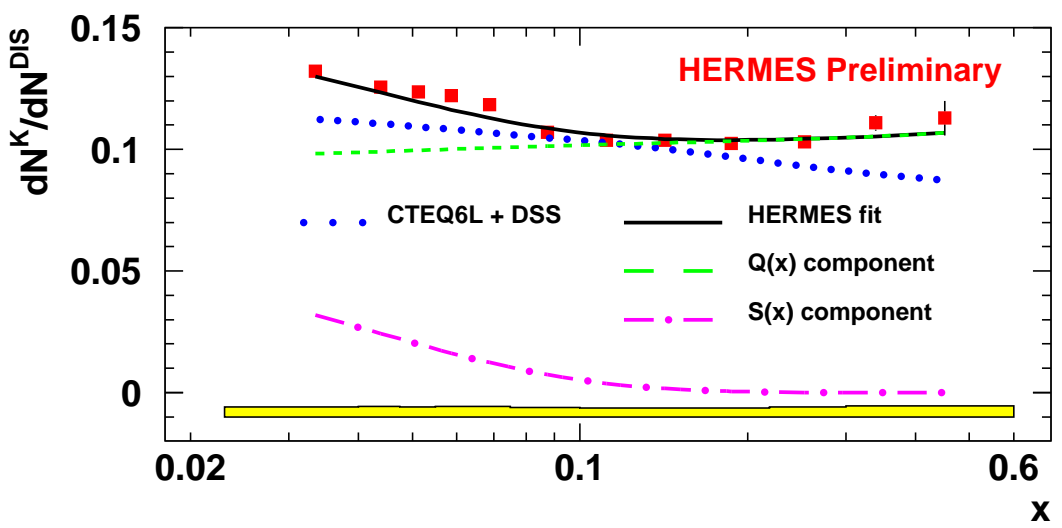

Figure 3: The multiplicity of charged kaons in SIDIS from a deuterium target, as a function of Bjorken $x$. The continuous curve is calculated from the curve in Fig. 2 together with the nonstrange contribution as extracted from the high $x$ multiplicity data (see text). The dashed (dash-dotted) curve is the nonstrange (strange) quark contribution to the multiplicity for this fit. The dotted curve is the LO prediction obtained with CTEQ6L PDFs and fragmentation functions from [3]. The band represents the systematic uncertainties. 


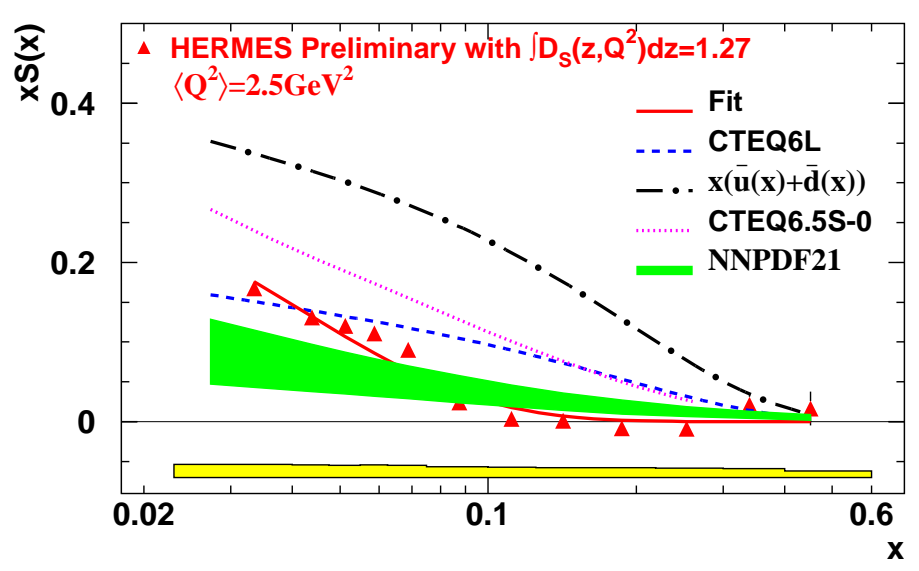

Figure 4: The strange parton distribution $x S(x)$ from the measured HERMES multiplicity for charged kaons evolved to $Q_{0}^{2}=2.5 \mathrm{GeV}^{2}$ assuming $\int \mathscr{D}_{S}^{K}(z) d z=1.27 \pm 0.13$. The solid curve is a 2-parameter fit for $S(x)=x^{-0.867} e^{-x / 0.033}(1-x) / 1.27$, the dashed curve gives $x S(x)$ from CTEQ6L, and the dot-dash curve is the sum of light antiquarks from CTEQ6L. The broad rising band is the $\pm 1 \sigma$ zone of allowed values predicted by the neural network methodology [5]. The band at the bottom represents the systematic uncertainties.

by the value of $\int \mathscr{D}_{S}^{K}(z) d z$ assumed. However, whatever the normalization, the shape of $x S(x)$ implied by the HERMES data is incompatible with $x S(x)$ from CTEQ6L as well as the assumption of an average of an isoscalar nonstrange sea. The absence of strength above $x \approx 0.1$ is clearly discrepant with CTEQ6L. Although the prediction of the neural network methodology based on the NNPDF2.1 LO parton set does not fit the shape of $\mathrm{S}(\mathrm{x})$ measured by HERMES , it does very well at describing the overall trend of $\mathrm{S}(\mathrm{x})$. This is in spite of preponderance in the data base of results from measurments at much higher energy scales.

\section{Acknowledgments}

We gratefully acknowledge the DESY management for its support, the staff at DESY and the collaborating institutions for their significant effort, and our national funding agencies and the EU FP7 (HadronPhysics2, Grant Agreement number 227431) for financial support.

\section{References}

[1] A. Airapetian, et al., Measurement of Parton Distributions of Strange Quarks in the Nucleon from Charged-Kaon Production in Deep-Inelastic Scattering on the Deuteron, Phys. Lett. B 666 (2008) 446 [arXiv:0803.2993].

[2] A. Airapetian, et al., Multiplicities of charged pions and kaons from semi-inclusive deep-inelastic scattering by the proton and the deuteron, Phys. Rev. D 87 (2013) 074029 [arXiv: 1212.5407 ].

[3] D. de Florian, R. Sassot and M. Stratmann, Global analysis of fragmentation functions for pions and kaons and thei r uncertainties, Phys. Rev. D 75 (2007) 114010 [hep-ph/ 0703242 ].

[4] A. Martin, et al., Scheme dependence, leading order and higher twist studies of MRST partons, Phys. Lett. B 443 (1998) 301 [hep-ph/9808371]. 
[5] R. Ball, et al., Unbiased global determination of parton distributions and their uncertainties at NNLO and at LO, Nucl. Phys. B 855 (2012) 153 [arXiv: 1107.2652 ]. 